\title{
III. Ueber das Sehen durch eine Kugel bei den Arabern;
}

von Eilhard Wiedemann.

1) Vor einiger Zeit hat Hr. Schellbach ${ }^{1}$ ) eine Notiz "Ueber eine unbekannte Eigenschaft der Convexlinsen" veröffentlicht. Die betreffende Eigenschaft war bereits den Arabern bekannt.

Die beiden arabischen Gelehrten, welche dieselbe, veranlasst durch das Studium des Regenbogens, durch geometrische Betrachtungen aufgefunden und dann experimentell constatirt haben, reihen sich würdig den anderen grossen arabischen Physikern, Al Birunî, Ibn al Haițam und Al Khâzinî an.

2) Der eine der beiden Forscher ist der Verfasser des Commentars zu der Optik des Ibn al Haitam in der Ley. dener Handschrift. ${ }^{2}$ ) Leider nennt er seinen Namen nicht, indess wissen wir, dass $\mathrm{Kam} \hat{a} l$ ed Dîn $\mathrm{Abu}$ al Hasan al Fôrisî einen Commentar zu der erwähnten Schrift verfasst hat, vielleicht ist dies derselbe, der uns hier vorliegt.

Unser Autor erzählt in der Einleitung zu seinem Werke, dass er sich schon lange dem Studium der Optik zugewandt habe, besonders hätten ihn diejenigen Erscheinungen interessirt, welche man beobachtet, wenn sich ein betrachteter Gegenstand hinter Wasser oder Glas befindet. Das, was über diese Fragen in Euklid's Optik mitgetheilt gewesen sei, habe ihm aber nicht genügt. Als er daher gehört habe, dass in persischen Bibliotheken ein Werk Ibn al Haitam's über diesen Gegenstand sich befände, sei er dahin gereist, habe dasselbe mit grossem Eifer studirt und mit einem Commentar versehen.

Bei seinen Arbeiten habe er sich reichlicher Hülfe und Unterstützung von Seiten eines Gelehrten in Persien zu er-

1) K. Schellbach, Zeitschr. für phys. u. chem. Unterricht 2 . p. 291.1889.

2) Vgl. E. Wiedemann, Wied. Ann. 39. p. 115.1890. 
freuen gehabt, dessen wissenschaftliche Bedeutung er selbst auf das höchste preist, nämlich von Kotb ed Dîn Ab̂̂ al Tanâ Mạ̣mûd ibn Masûd al Schîrasî (1236-1311). $\left.{ }^{1}\right)$

3) An die abgekürzte Redaction der Optik des Ibn al Haitam und den Commentar schliessen sich zunächst wei: tere Ausführungen einer Reihe von Aufgaben, welche sich auf die Brechung des Lichtes beziehen, so über die Gestalt in der ein Kreis unter Wasser erscheint etc.

Daran reiht sich die Einleitung zur Untersuchung der Himmelszeichen (Regenbogen, Halo etc.), deren Inhalt im wesentlichen der folgende ist:

$\mathrm{Zu}$ den wunderbarsten Werken Gottes gehören die Himmelszeichen, vor allem der Halo und der Regenbogen. Frühere Gelehrte haben ihren Scharfsinn angestrengt, um die Ursachen der Entstehung derselben zu ergründen, und haben auch eine Reihe von Erklärungen geliefert, welche wohl theoretisch ganz richtig sind, aber nicht mit den thatsächlichen Verhältnissen übereinstimmen. „Da suchte ich Hülfe bei einem rechten Helfer, der den Schleier fortnehmen sollte, und er that dies und eröffnete mir eine Thür, durch welche wir eine Lösung der Sache erhoffen konnten. Ich habe dieselbe nun klar gelegt. Zunächst will ich aber die Ansichten der früheren Gelehrten wiedergeben und zwar des Ibn al Haitam ${ }^{2}$ ) und des Aricenna.“3)

Der Helfer ist jedenfalls der oben erwähnte Kotb ed Dîn al T́anâ. Er hat also die Ideen zu den Entwickelungen yeliefert, welche $K$ amâl ed Dîn dann weiter ausgeführt hat. Wie viel Verdienst der eine und wie viel der andere Gelehrte hat, lässt sich jetzt nicht mehr angeben.

1) Näheres über die Einleitung und die Lebensverhältnisse der beiden Gelehrten denke ich an einem anderen Orte mitzutheilen.

2) Schriften des Ibn al Haitam, die hier in Frage kommen, sind sein Auszug der Meteorologie des Aristoteles (Ibn Abi Useibia ed A. Müller p. 97, Z. 18 v. oben), seine Schrift „Ueber den Regenbogen und den Halo" (ibid. Z. 6 v. unten).

3) Vgl. Wüstenfeld, Geschichte der arabischen Aerzte Nr. 128. Schriften des Avicenna, die sich mit Meteorologie beschäftigen, resp. Theile enthalten, die dies thun, sind Kitab al Schafầ (liber sanationis), der Auszug aus demselben Kitâb al Nagât, und wahrscheinlich wohl 
Nun folgen die Anschauungen Ibn al Haitam's über die Himmelszeichen, die, wie schon die Figuren zeigen, sich eng an diejenigen des Aristoteles anschliessen und die, wie $\mathrm{Kam}$ âl ed Dîn angibt, einer im Jahre 419 d. H. im Monate Rageb eigenhändig von al Haițam geschriebenen Arbeit entnommen sind. Ganz kurz reihen sich die Entwickelungen Avicennas' an, entnommen der Physik in seiner Schafâa, alles stets von einem Commentar begleitet.

4) Im Anschluss an Avicennas' Ansichten weist Ka mâl ed Dîn nun die Unrichtigkeit der Ansichten der früheren Gelehrten nach. Durch blosse Reflexion an den Wassertropfen könne kein Regenbogen entstehen, denn wenn an der Oberfiäche einer Glaskugel das Bild eines Lichtes reflectirt wird, so zeige sich wohl ein $\mathrm{Kreis,}$ der in seinen verschiedenen Theilen verschieden hell sei, aber diese Reflexion könne nie Veranlassung geben zu dem Auftreten zweier räumlich getrennter Regenbögen, bei denen die Farben auch an verschiedenen Stellen liegen, ebensowenig lasse sich der Halo aus ihr erklären. „Lässt sich die Sache aber nicht so erklären, so muss man sie entweder von demselben Grundgedanken ausgehend, frei behandeln oder aber einen ganz neuen Weg einschlagen. Gott hat uns nun den Weg zu einer freien Behandlung gewiesen und wir hoffen, dass uns ein anderer Weg in derselben genügt."

Aus dem Folgenden geht hervor, dass die Reflexion an den Wassertropfen beibehalten wird, aber zu der Reflexion an der Oberfläche noch die Brechungen in die Tropfen und die Reflexionen im Inneren derselben herbeigezogen werden, gerade wie bei unserer modernen Erklärung.

Hierauf wendet sich der Verfasser zu der Brechung des Lichtes in Kugeln:

5) Das erste Kapitel enthält eine Revision der Abhandlung über die Brennkugel von Ibn al Haitam.

Einiges aus dieser Schrift habe ich schon früher mitgetheilt. ${ }^{1}$ ) Eine vollständige Uebersetzung soll an anderem

auch seine Commentare zu Aristoteles' Schriften. Eine Schrift, deren Titel direct auf den Halo und den Regenbogen sich bezöge, habe ich nicht finden können.

1) E. Wied emann, Wied. Ann. 7. p. 679. 1879. 
Orte erscheinen. Ich bemerke hier nur noch, dass bei den Ableitungen auf das ausgiebigste Gebrauch gemacht wird von dem Satz: Dass bei einer Kugel der Brechungswinkel im Glase an der Eintrittsstelle gleich dem Einfallswinkel im Glase an der Austrittsstelle ist, oder dass daher Einfallswinkel und Austrittswinkel in der Luft einander gleich sind. (Kugelfächen und planparallele Flächen verhalten sich also in dieser Hinsicht einem sie durchsetzenden Lichtstrahl gegenüber gleich).

6) Das zweite Kapitel über das Sehen der Gegenstände durch eine glatte Kugel interessirt uns hier speciell.

Ich gebe nur einen ganz kurzen Auszug aus den arabischen Entwickelungen. Dieselben sind äusserst weitschweifig, wodurch das Studium sehr erschwert wird. Die Handschrift ist eine gute Gelehrtenhand, sehr correct, aber, wie schon die Verfasser des Leydener Handschriftencataloges sagen, "Charactere minutissimo et interdum lectu difficiliori exaratae". Indess dürfte die Mühe, die ich auf dieselbe verwandt, durch das Ergebniss belohnt sein.

Das zweite Kapitel zerfällt in vier Abschnitte.

7) Der erste behandelt das Sehen durch eine Kugel. Das Auge erhält von den Gegenständen durch die Bilder Kenntniss, welche sich längs der Strahlen fortpflanzen. Wenn das von einem Punkt auf geraden Linien fortschreitende Licht nach einer anderen Richtung reflectirt oder gebrochen wird, so wird der Punkt, nach dem die Richtung geht, als leuchtend angesehen. Ist ein Lichtstrahl $A B$ ursprünglich in dem Punkt $B$ nach $B C$ reflectirt oder nach $B D$ gebrochen und bewegt der Lichtstrahl sich dann in der Richtung $C B$ oder $D B$ nach dem Punkt $B$, so wird er in der Richtung $B A$ weiter schreiten. Dasselbe gilt, wenn mehrere Brechungen oder Reflexionen eintreten. Ist dies aber der Fall, so können wir den Weg von Gegenstand zu Auge auch umgekehrt durchlaufen und entweder den Gegenstand als leuchtend ansehen oder aber das Auge. ${ }^{1}$ )

Strahlen, die von dem Mittelpunkt der Sonne nach einer durchsichtigen Kugel gehen, werden zweimal gebrochen, ein

1) Dieser Satz rührt also nicht von Witelo (Poggendorff, Geschichte der Physik p. 91), sondern von den Arabern her. 
erstes mal aus der Luft in dieselbe, ein zweites mal aus derselben in die Luft.

Stellt man vor das Auge eine durchsichtige glatte Kugel, so ist das Gebilde zwischen dem Auge und dem ihm zunächst gelegenen Theile der Kugel eine Kegelfläche. Ihre Axe ist die Verbindungslinie der Mittelpunkte von Auge und Kugel. Der der Axe entsprechende Strahl geht in gerader Richtung ungebrochen fort. Die anderen Strahlen werden an der Kugel gebrochen. Se weiter man sich von dem Pol (dem Schnittpunkt der Axe mit der Kugel) entfernt, um so grösser werden die Einfallswinkel. Der Einfallswinkel von $90^{\circ}$ liegt am Ende des Schnittes. Ist nun der Abstand des Auges so gross, wie der des Sonnenmittelpunktes, so gestalten sich die Brechungen nach früheren Entwicklungen (in der Schrift über die Brennkugel) folgendermassen. DieStrahlen, welche auf einen Punkt der Kugel unter dem Einfallswinkel von $40^{\circ}$ auftreffen, gelangen nach der Brechung auf einen Punkt der Kugel, auf den auch diejenigen Strahlen treffen, die dem Einfallswinkel von $50^{\circ}$ entsprechen. Ebenso gelten für Strahlen mit Einfallswinkeln unter $40^{\circ}$ und über $50^{\circ}$, dieselben Betrachtungen, wie für die Sonnenstrahlen. Alle Strahlen, die auf die Kugel treffen, werden infolge der Glätte reflectirt und infolge der Durchsichtigkeit gebrochen. Treffen sie auf die Kugelfläche auf der anderen Seite, so werden sie von neuem reflectirt und gebrochen.

Wir zeichnen eine der Brechungsebenen, welche die Kugel in einem Kreise schneiden, wir ziehen vom Auge Strahlen nach den Bögen, deren Enden Einfallswinkeln von 10,20 bis $90^{\circ}$ entsprechen. Solcher Linien sind es 9, mit der Axe 10. Wir nennen die 5 ersten die erste und die übrigen die $\mathrm{zweite}$ Schaar.

Alle diese Strahlen werden nach der Brechung auf einem Stück der Kugel vereint, das dem ersten gerade gegenüber liegt, dessen $\mathrm{Pol}$ dem $\mathrm{Pol}$ des ersten gegenüber liegt und dessen Umfang eine Kreistäche ist. Zwischen letzterer und dem Pol liegt ein Zehntel eines grössten Kreises der Kugel. Wir nennen diese Kreisfläche die Vereinigungsfläche.

Wir betrachten zunächst die erste Schaar der Strahlen. Sie treffen die Vereinigungsfläche in ihrer ursprünglichen 
Reihenfolge auf, d. h. die von der Axe entfernteren werden nach einem von der Axe weiter entfernten Punkt hin gebrochen.

Die Strahlen der zweiten Schaar treffen in einer der ursprünglichen Reihenfolge entgegengesetzten auf, d. h. die von der Axe entfernteren werden nach Punkten, die der Axe näher liegen, gebrochen. Es ist gezeigt worden, dass alle Strahlen die Axe ausserhalb der Kugel treffen, mag nun die Kugel an dem zugewandten Ende vollkommen sein oder nicht (d.h. mag sie dort von einer Kugeloberfläche oder einer Ebene begrenzt sein).

Der der Axe nähere Strahl trifft dieselbe in einem Punkt, welcher weiter von dem Pol des Vereinigungsortes absteht. Ist die Kugel vollkommen, so werden die Strahlen ein zweites mal gebrochen und ihr Schnittpunkt mit der Axe liegt näher an dem Pol.

8) Im zweiten Abschnitt wird zunächst gezeigt, dass, wenn das Auge sich näher an der Kugel befindet, es einen grösseren Theil der aligewandten Fläche derselben sieht, als wenn es weiter davon entfernt ist.

Dies wird folgendermassen bewiesen. (Fig. 1.)

Das Auge befinde sich zunächst in $l$, dann gelangen die von dem Auge ausgehend gedachten Strahlen nach rg, und

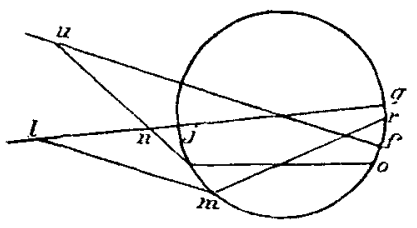

Fig. 1. $r g$ wird von dem Auge gesehen. Drehen wir nun die ganze Figur um den Mittelpunkt des Kreises, sodass $l$ nach $u$ kommt, $g$ nach $f, r$ nach $o$, so sieht das Auge jetzt das Stück of. Rücken wir das Auge nach $n$, so ist aus der Construction klar, dass es dann das ganze Stück go übersieht.

Der Absatz schliesst: „Dies habe ich durch den Versuch bewiesen. Ich habe das Auge der Kugel sehr genähert, und die Kugel auf ein beschriebenes Blatt Papier gelegt, da habe ich von der Schrift mehr wahrgenommen, als wenn das Auge von der Kugel weiter entfernt war."

9) Anschliessend hieran wird eine Terminologie für die Räume eingeführt, die von den Strahlen der ersten und der 
$z w e i t e n$ Schaar erfüllt sind: sie sind charakterisirt durch die Reihenfolge, in der die Strahlen auf den Vereinigungsort treffen (Fig. 2). ${ }^{1}$ )

Weiter wird darauf aufmerksam gemacht, dass durch die Brechung das Licht geschwächt wird, und zwar um so stärker, je stärker die Brechung ist ${ }^{2}$, daraus folgt dann, dass in dem inneren Kegel die Wärme grösser ist, als in dem äusseren, und zwar um so grösser, je näher man der Axe sich befindet.

10) Der Verfasser verfolgt nun die Strahlen genauer, welche im Abstand von 50 Grad vom $P_{0 l}$ auf die Kugel fallen. Sie werden nach th und $k h$ gebrochen, dadurch zerfällt die Ebene der Zeichnung in mehrere Abschnitte, und bei einer Rotation um ihre Axe auch der Raum. Jenachdem ein Körper sich in dem

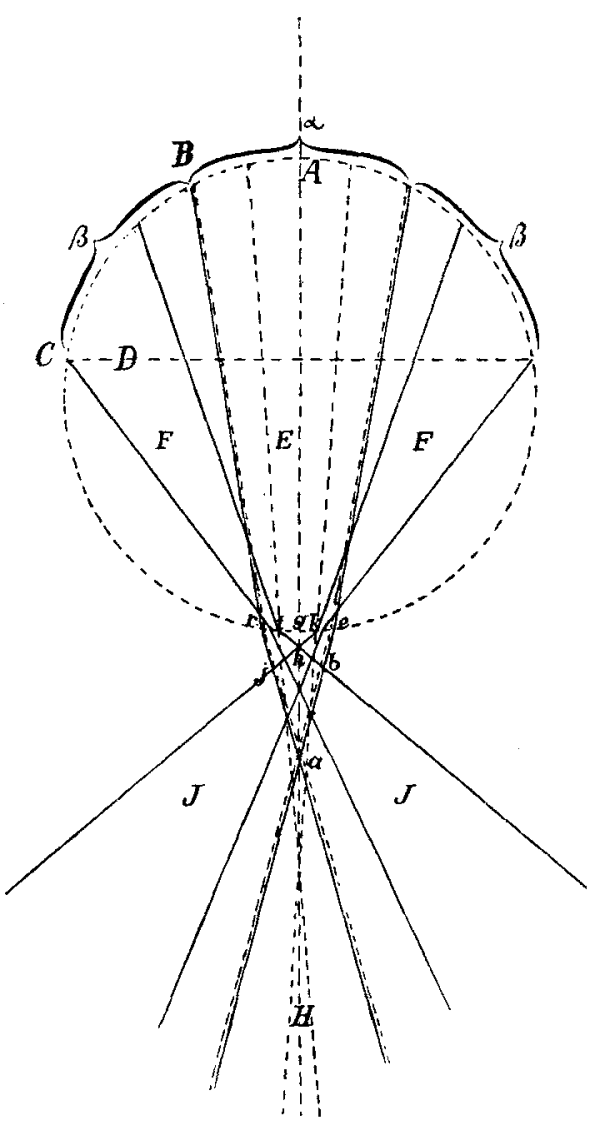

Fig. 2.

1) Die Figur trägt folgende Bezeichnungen: $A$ Pol deg zugewandten Stückes, $B$ Grenze der beiden Kegel, $C$ Tangentialpunkt, er ist die Grenze des zugewandten Stückes, $D$ Sehne des zugewandten Stückes, $E$ Mittelkegel, $F F$ Hohlraum, $H$ umgekehrter Mittelkegel, $J J$ umgekehrter Hohlraum. Die durch den Bogen $\alpha$ zusammengefassten Strahlen bilden die erste, die durch die Bögen $\beta \beta$ zusammengefassten die zweite Schaar, re ist der Vereinigungsort. Die kleinen Buchstaben stehen im Original, die nicht punktirten Linien sind im Original schwarz, die punktirten roth.

2) Dieser Satz rührt also nicht von Wîtelo her. Poggendorff, Geschichte p. 91 . 
Theil $t h k, a j h b$ oder den Theilen $k h b e$ und thjr befindet, oder in zweien zugleich, muss er, durch die Kugel betrachtet, verschiedenartige Bilder liefern, aufrechte, verkehrte, oder verkehrte und aufrechte zugleich, indem sich bei der Brechung die Reihenfolge der Strahlen von innen nach aussen umgekehrt, nicht umkehrt, oder für einen Theil umkehrt, für einen anderen nicht.

Dies wird durch zwei Versuche geprüft.

a) Der betreffende Gegenstand sei klein, etwa ein Kreis. Wir halbiren seinen Durchmesser und machen die eine Hälfte roth, die andere schwarz, (Fig. $3_{\mathbf{a}}$ statt roth und schwarz in der Figur horizontal und vertical gestrichelt), dann bringen wir seinen Mittelpunkt nach dem Punkt $h$, er erscheint grösser als er wirklich ist und aufrecht. Man entfernt ihn langsam von der Kugel längs der Axe. Mit zunehmender Entfernung nimmt seine Grösse zu, bis man einen Ring von denselben Farben wie er selbst sieht. (Fig. $\left.3_{b}\right)^{1}$ ), d. h. der Ring erscheint aus einem schwarzen und einem

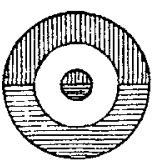

Fig. 3b. Fig. 3a. rothen Theil zusammengesetzt. Die beiden Ringe stossen unmittelbar aneinander. Bei zunehmendem Abstand nimmt die Breite des Ringes langsam zu, sein Umfang wird aber kleiner, der Kreis im Inneren des Ringes wächst und nimmt durch Berührung mit dem Kreis selbst die Grestalt des Kreises an. Hierbei ist der Gegenstand bis an die Spitze des inneren Kegels nach der zweiten Brechung gelangt, tritt er in den Bereich des umgekehrten Kegels ein, so wird das Bild des Kreises verkehrt. Es wird kleiner und kleiner, kleiner als es vorher war und so geht es fort, bis es den Blicken entschwunden ist.

b) Es seien zwei Kreise (Fig. $4_{a}$ ) um einen Mittelpunkt gegeben, von denen der grössere roth und der kleinere schwarz und voll ist. Man stellt sie so, dass die Axe, welche durch ihren Mittelpunkt geht, senkrecht auf ihnen steht. Befinden sie sich in dem umgekehrten Kegel ausserhalb des Brennkegels, so sieht man zwei Kreise in der richtigen Lage,

1) Innerhalb des Ringes bleibt der Kreis selbst sichtbar. Die Figuren stehen nicht im arabischen Text. 
d. h. der grössere ist roth und der kleinere schwarz. Nähert man sie der Kugel, so werden sie fortwährend grösser. Kommt man so nahe, dass der Umkreis des kleinen mit dem Brennpunkt zusammenfällt, so sieht man einen schwarzen Kreis in dem Inneren der kleineren schwarzen auftreten. (Dies ist der Versuch des Hrn. Schellbach.) Die beiden Kreise weichen auseinander, der grössere nach dem Rande, der kleinere nach der

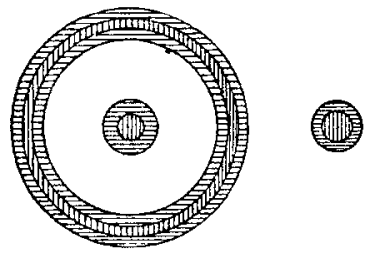

Fig. 4b.

Fig. 4a. Mitte, bis dass der Umkreis des grösseren mit der Begrenzung der Kugel zusammenfällt, und er erscheint als ein rother Kreis.

In einem bestimmten Stadium haben wir von aussen nach innen, wenn wir von den nicht gefärbten Partien absehen, einen rothen, einen schwarzen, einen rothen, einen rothen, einen schwarzen Kreis (wie dies Fig. $4_{b}$, die, wie $4_{a}$, nicht im arabischen Text steht, erläutert). Beim weiteren Annähern verschwindet einer der drei Kreise nach dem andern, indem sie über die Begrenzung der Kugel herausrücken und es bleiben nur die beiden inneren, sobald die Begrenzung des grösseren rothen Kreises innerhalb des Raumes thk fällt. In einer Bemerkung wird noch beigefügt, dass demnach durch eine Kugel das rechte links erscheint und umgekehrt, und dass dieses durch das Experiment bewiesen sei für leuchtende Körper und für gefärbte.

Sehr eingehend erörtert und durch die beistehende Figur 5 erläutert (die punktirten Linien sind im Original roth, die nicht punktirten schwarz) wird der Gang der Strahlen, die von d ausgehen, bei einmaliger Brechung in die Kugel, und a) bei einmaliger und b) bei zweimaliger Reflexion innerhalb derselben und zweiter Brechung aus der Kugel in die Luft. Zur Untersuchung werden gewählt, ein Strahl (1), der dem Mittelkegel angehört, der Grenzstrahl (2) des Mittelkegels, der Grenzstrahl des Hohlraumes (4) und ein beliebiger Strahl desselben (3). Es wird gezeigt, wie die ursprünglich rechts gelegenen Strahlen nach der ersten Reflexion nach links kommen und umgekehrt. 
Nur für die nicht punktirten Strahlen ist der Gang auch nach der zweiten Reflexion gezeichnet. Hieran schliessen sich die folgenden Beobachtungen an:

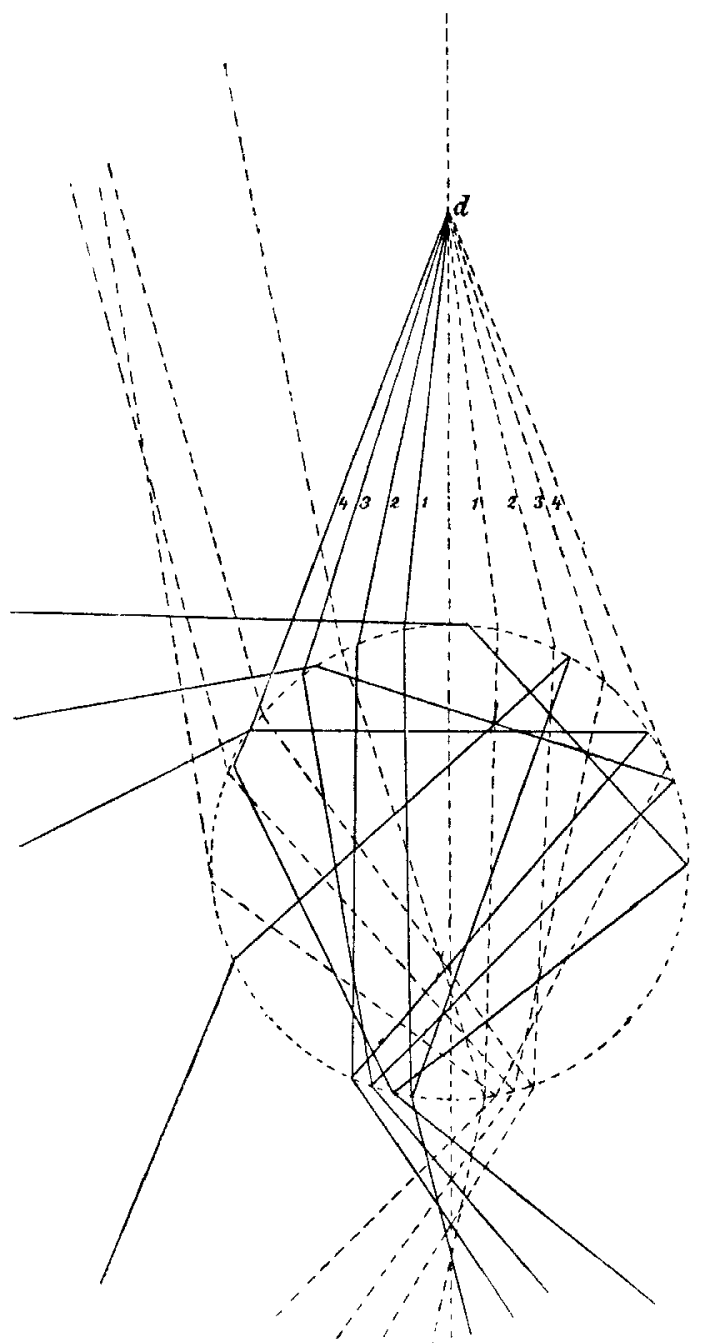

Fig. 5.

a) Beobachtung der nach einer Reflexion gebrochenen Strahlen. Der Beobachter geht in ein verfinstertes Haus, in dem ein Loch sich befindet, durch welches das Sonnen- 
licht einfällt. In das Sonnenlicht hält er eine Glaskugel und legt auf die Erde, da, wo das Licht auftritt einen schwarzen Körper. Dann umhüllt er die eine Hälfte der Kugel mit einem dichten weissen Körper und blickt auf die Seite desselben, welche der Kugel zuliegt. Dort sieht er einen farbigen Bogen, dessen Mittelpunkt auf der Seite zwischen dem Mittelpunkt der Kugel und der Sonne liegt. Der innere Theil des Bogens ist heller als der äussere. Der Bogen ist ein Stück der Basis des Kegels, welcher die nach einmaliger Reflexion gebrochenen Stahlen enthält. Nähert man den dichten Körper der Glaskugel, so wird der Kreisbogen kleiner, seine Breite geringer und die Farben undeutlicher.

Ueber die Farben verbreitet sich der Verfasser an einer anderen Stelle, wir gehen hier nicht darauf ein.

b) Beobachtung der gebrochenen und zweimal reflectirten Strahlen. Diese geschieht in ganz derselben Weise, nur wird sie mit dem Auge direct vorgenommen, „denn beobachtet man in derselben Weise, wie bei der Brechung nach einmaliger Reflexion, so findet man die Sache wie bei directer Beobachtung, nur ist die Beobachtung wegen der geringeren Lichtstärke schwieriger, gerade ebenso wie es bei der Brechung nach einmaliger Reflexion der Fall ist, wenn die Lichtquelle nicht so kräftig wie die Sonne ist, sondern eine Lampe oder der Mond ist oder die Kugel weit absteht".

Der Beobachter sieht in dem Brennkegel sehr helles und ungefärbtes Licht, das gebrochene Bild nach einmaliger Reflexion ist etwas gefärbt, nach zweimaliger Reflexion stärker gefärbt.

12) In dem diesem Abschnitte folgenden werden dann die gewonnen Resultate auf die meteorologischen Erscheinungen der Himmelszeichen angewandt, dabei auch Reflexionen und Brechungen beim Vorhandensein mehrerer Kugeln besprochen. Den Hauptregenbogen erklären unsere Gelehrten, gerade wie wir heute, durch einmalíge, den Nebenregenbogen durch zweimalige Reflexion im Inneren der Tropfen.

13) Dann folgt in der Handschrift noch eine Abhandlung über Schatten, eine über Sonnenfinsternisse, ein Auszug aus der Abhandlung Ibn al Haitam's über das Licht. ${ }^{1}$ )

3) Vgl. E. Wiedemann, Wied. Ann. 20. p. 337. 1883. 
14. Einen Versuch, bei dem ein schwarzer Punkt durch eine Glaskugel betrachtet als Ring erscheint, beschreibt auch Ibn al Haitam in seiner Optik ${ }^{1}$ ) und ihm folgend Witelo. ${ }^{2}$ ) Eine Vergleichung der Erklärung der Erscheinungen bei Ibn al Haițam und Kamâl ed Dîn zeigt, wie weit der letztere dem ersteren voraus war.

15) Die im obigen mitgetheilten Untersuchungen dürften ein glänzendes Zeugniss für die Thätigkeit arabischer For. scher auf dem physikalischen Gebiete ablegen. Sie sind doppelt interessant deshalb, weil wir gar keine Andeutung haben, dass die Griechen oder Römer sich wissenschaftlich mit der Brechung an gekrümmten Flächen befasst haben, während bei der Erörterung der Erscheinungen an den Hohlspiegeln sich den Arabern manche Anknüpfungspunkte im Alterthum boten.

Inwieweit Wîtelo, Theodoricus Saxonicus, de Dominis u. s. f., die im Mittelalter Erklärungen des Regenbogens aufstellten und dabei abweichend von Aristoteles Reflexionen im Inneren der Tropfen zur Erklärung herbeizogen, durch die arabischen Entdeckungen beeinflusst waren, lässt sich auf Grund des vorliegenden Materials noch nicht entscheiden; dass die abendländischen Gelehrten auch hier gewiss vieles den Arabern zu verdanken haben, dürfte kaum zweifelhaft sein.

Erlangen, im Januar 1890.

1) Opticae ed. Risner lib. 7. pro. 49. p. 277.

2) Wîtelo, lib. 10. prop. 43. p. 440. 